\title{
High Heat Load Performance of an Inclined Crystal Monochromator with Liquid Gallium Cooling on the CHESS-ANL Undulator*
}

\author{
A.T. Macrander, W.K. Lee, R.K. Smither, \\ D.M. Mills, S. Rogers, and A. Khounsary \\ Advanced Photon Source \\ Argonne National Laboratory \\ 9700 South Cass Avenue \\ Argonne, IL 60439
}

$\mathrm{ANE} / \mathrm{CP}-74078$

DE92 004131

November, 1991

cz

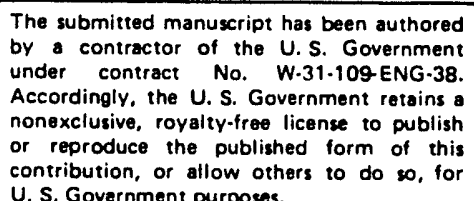

*This work supported by the U.S. Department of Energy, BES-Materials Sciences, under contract no. W-31-109-ENG-38

\section{DISCLAIMER}

This report was prepared as an account of work sponsored by an agency of the United States Government. Neither the United States Government nor any agency thereof, nor any of their employees, makes any warranty, express or implied, or assumes any legal liability or responsibility for the accuracy, completeness, or usefulness of any information, apparatus, product, or process disclosed, or represents that its use would not infringe privately owned rights. Reference herein to any specific commercial product, process, or service by trade name, trademark, manufacturer, or otherwise does not necessarily constitute or imply its endorsement, recommendation, or favoring by the United States Government or any agency thereof. The views and opinions of authors expressed herein do not necessarily state or reflect those of the United States Government or any agency thereof.
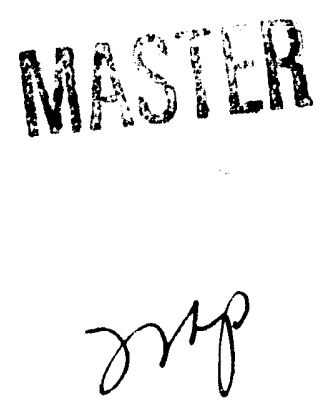

SRI
Baton Rouge
$10 / 31$
$(N \mid M)$

\title{
HIGH HEAT LOAD PERFORMANCE OF AN INCLINED- CRYSTAL MONOCHROMATOR WITH LIQUID-GALLIUM COOLING ON THE CHESS-ANL UNDULATOR
}

\author{
A.T. Macrander, W.K. Lee, R.K. Smither, D.M. Mills, C.S. Rogers, and A.M. Khounsary
}

Advanced Photon Source, Argonne National Laboratory, Argonne, IL 60439

\begin{abstract}
Results for the performance of a novel double crystal monochromator subjected to high heat loads from an APS-prototype undulator at the Cornell High Energy Synchrotron Source (CHESS) are presented. The monochromator was designed to achieve symmetric diffraction from Bragg planes that are inclined relative to the surface. The angle of incidence can be made small in the inclined arrangement thereby lowering the power density incident on the surface of the crystal. Both crystals had (111) oriented surfaces and were arranged such that the beam was diffracted from the (111) planes at $5 \mathrm{KeV}$. Rocking curves with minimal distortion were obtained at a ring electron current of $96 \mathrm{~mA}$. This corresponded to 379 Watts total power and a peak power density of $48 \mathrm{Watts} / \mathrm{mm}^{2}$ transverse to the incident beam. These results are compared to data obtained from the same crystals in the standard arrangement (diffracting planes parallel to surface). The footprint area in the inclined case was three times that of the standard case. We also obtained rocking curve data for the $\{333\}$ reflection at $15 \mathrm{KeV}$ for both the standard and inclined cases, and these data also showed a minimal distortion for the inclined case. In addition, thermal data were obtained via infrared pyrometry. Not only the diffraction data but also the thermal data revealed a dramatically improved performance for the inclined-crystal case.

\section{Introduction}

The thermal benefits of enlarging a beam foatprint on the first crystal of monochromators for synchrotron radiation have been recognized for several years ${ }^{1}$. A larger footprint serves to lower the power density incident on the crystal face. Monochromator designs involving small angles of incidence are particularly well suited for undulator radiation because undulator beams are small. For undulator radiation, significant spreading of the beam footprint can be accomplished with moderately large crystals, that is, unreasonably large crystals are not required.

A simple way in which to accomplish spreading of the footprint is to diffract from planes that are not parallel to the surface. This is often done with the surface normal constrained to lie in the diffraction plane. The angle of incidence and the angle of exit relative to the surface are then not equal, and the angle of incidence can be made quite small by cutting a crystal such that the angle of the planes relative to the surface is slightly less than the Bragg angle. However, the tunability range is then severely restricted because the beam must impinge at angles larger than the critical angle for total external reflection.
\end{abstract}


However, tunability is not necessarily sacrificed. Tuning can also be accomplisheci by rotating the reciprocal lattice vector about the surface normal ${ }^{2,3}$. An appealling aspect of this suggested monochromator design is that the angle of incidence is the same at all energies so that the same footprint spreading factor is in effect. However, the beam will walk both vertically and horizontally as such a monochromator is tuned from one energy to another. The complications resulting from two perpendicular beamwalks in such an asymmetric reflection monochromator caused us to choose another design.

The alternative diffraction geometry that we chose does not involve an additional beamwalk and has a number of other advantages as well. A preliminary report of the results presented here was made on July $20,1991^{4}$. The new geometry was proposed and was given the name 'inclined' by Khounsary. The same geometry has been proposed by $\mathrm{Hrdy}^{8}$. We have built and tested an inclined crystal monochromator at the Cornell High Energy Synchrotron Source (CHESS) using first harmonic radiation from the CHESS-ANL undulator? . Results for this monochromator are described below.

\section{Inclined Crystal Monochromator}

In the inclined crystal arrangement, shown in Fig.1, diffraction occurs from planes which are not parallel to the surface. However, both the incident and diffracted beams make the same angle with respect to the surface normal, and, therefore, the diffraction is symmetric. There are several important features to this design.

The monochromator can be operated in a manner identical to that for a standard monochromator, that is, with a fixed plane of diffraction. In the inclined arrangement, the Bragg planes themselves are positioned just as for a standard monochromator. (For convenience, we will henceforth refer to the standard situation as the 'flat-crystals' case which corresponds to an inclination angle, $\beta$, [see Fig.1] equal to zero .) If one keeps in mind only the Bragg planes and ignores the surface, then the flat and inclined cases are alike.

For an aligned inclined monochromator the diffraction is symmetric, which implies that the factor known as $b$ in dynamical diffraction $n^{8}, 9$ equals -1 . This factor is defined as $s_{0} \cdot n / s_{H} \cdot n$, which is the ratio of the direction cosines of the incident $\left(s_{0}\right)$ and diffracted $\left(\mathbf{s}_{\mathrm{H}}\right)$ beams with respect to $n$, the inward surface normal. Darwin widths are modified by a factor of $|\mathrm{b}|^{1 / 2}$. ( The asymmetry factor deviates from the value -1 if an inclined monochromator is misaligned ${ }^{10}$.) The inclined arrangement does not impose constraints beyond those present in the flat case due to modified Darwin widths. For example, the required angular resolution of goniometers used to set the Bragg angles is the same for the flat and inclined cases.

The tuning range of an inclined crystal is not restricted beyond that of a flat crystal. Bragg angles at high energies become quite small in both cases, but the critical angle for total external reflection is never reached in either case because the critical angle also decreases with increasing energy. For all reasonable inclination angles, the critical angle is always less than the angle of incidence as one goes to 
higher energies. In the inclined case, the angle of incidence, $\phi$, is not equal to the Bragg angle but instead satisfies,

$$
\sin \phi=\cos \beta \sin \theta,
$$

where $\theta$ is the Bragg angle. Provided $\beta$ is not chosen larger than $89.1^{\circ}$ for $\mathrm{Si}(111)$ the critical angle will not be reached at any energy.

The footprint of the beam on the crystal surface was detailed in ref. 5 and is shown in Fig. 2. The length labeled $c$ in this figure vanishes in the flat case but is made large in the inclined case by choosing large values of $\beta$. Each long edge of the parallelogram shaped footprint lies in a horizontal plane and has a length that is roughly equal to $c$ when $\beta$ is very large. For large $\beta$, the footprint is a long thin stripe. Figure 2 is shown for a vertical diffraction plane, and, in this case, the length of the stripe depends primarily on the horizontal width, $w$, of the beam and only weakly on the vertical height, $h$.

The area of the beam footprint is given by $A(\sin \phi)^{-1}$, where $A$ is the transverse area of the beam, not only in the flat case but also in the inclined case. The simple spreading factor of $(\sin \phi)^{-1}$ applies also in the inclined case even though the shape of the footprint on the crystal is not rectangular. The enhanced spreading, i.e., amplification, of the footprint in the inclined case compared to the flat case is, consequently, given by $(\cos \beta)^{-1}$ (see Eq. 1 ) which is three for $\beta=70.529^{\circ}$.

Another benefit of the inclined geometry for high heat load applications is that the angle between the surface normal and the diffraction plane is $\beta$ which implies that only a fraction of the thermal distortion will lie in the diffraction plane. Consequently, we expect that only a portion of any deformation resulting from a high heat load should contribute to the rocking curve width.

III. Experimental Details

A. Undulator Source and Beam Dimensions

Experiments were conducted at CHESS with the undulator 7 placed in the Cornell Electron Storage Ring (CESR). With CESR in dedicated operation at 5.433 $\mathrm{GeV}$, the undulator first harmonic occurred at $5.14 \mathrm{KeV}$ with a gap of $1.7 \mathrm{~cm}$. Calculated phase space parameters for CESR are given in Table I.

Table I. CESR Parameters at Undulator Center.

\begin{tabular}{cccccc}
\hline $\begin{array}{c}\varepsilon_{x} \\
\text { nm-rad }\end{array}$ & $\begin{array}{c}\varepsilon_{y} \\
\text { nm-rad }\end{array}$ & $\begin{array}{c}\sigma_{x} \\
\mathrm{~mm}\end{array}$ & $\begin{array}{c}\sigma_{x}^{\prime} \\
\mathrm{mrad}\end{array}$ & $\begin{array}{c}\sigma_{y} \\
\mathrm{~mm}\end{array}$ & $\begin{array}{c}\sigma_{y^{\prime}} \\
\mathrm{mrad}\end{array}$ \\
\hline 67 & 1.3 & 1.16 & 0.056 & 0.076 & 0.017 \\
\hline
\end{tabular}


The first crystal in our double-crystal arrangement was placed $18 \mathrm{~m}$ from the undulator source. The beam dimensions for the frequency-integrated power," which are calculated from the values in Table I, are given in Table II.

Table II. Transverse Beam Dimensions at $18 \mathrm{~m}$.

\begin{tabular}{lcc}
\hline & $\begin{array}{c}\text { horizontal }(x) \\
\mathrm{mm}\end{array}$ & $\begin{array}{c}\text { vertical }(y) \\
\mathrm{mm}\end{array}$ \\
\hline FWHM & 4.6 & 2.0 \\
$1.25 / \gamma$ & 8.0 & 4.7 \\
\hline
\end{tabular}

This corresponds to $10 \%$ of peak value in both the vertical and horizontal directions.

Calorimetry was performed at $49.4 \mathrm{~mA}$ ring current, and we measured a value of 195 Watts total power. The power density profile transverse to the beam has been calculated based on these results and is shown in Fig. 3 for $100 \mathrm{~mA}$ ring current, i.e., for 395 Watts total power. The peak value is 50 Watts $/ \mathrm{mm}^{2}$ at $100 \mathrm{~mA}$.

\section{B. Monochromator Details}

We chose the (111) reflection from a (111) silicon crystal for the inclined case. The value of $\beta$ for this case is $70.529^{\circ}$. Because the surface of the crystals was (111) oriented, we could also make our measurements in both the flat and inclined geometry with the same crystals, thus allowing us to compare directly the improvement due to the inclination. The first crystal is shown in Fig. 4 and is identical to one used in heat load experiments for the permanent magnet wiggler at $\mathrm{CHESS}^{12}$. The crystal was cooled with liquid gallium ${ }^{1,^{13}}$ which was pumped through slots having a rectangular cross section. The slots were $1.00 \mathrm{~mm}$ wide and $4.15 \mathrm{~mm}$ deep. The thickness of the ribs between the slots was $0.55 \mathrm{~mm}$, and the distance between the slots and the diffraction surface was $0.75 \mathrm{~mm}$. This so called 'slotted' crystal was made in two pieces. The slots were cut with a diamond wheel in one piece of silicon that was then cemented to a second piece of silicon. The cemented crystal assembly was then mounted on a stainless steel base and sealed with ' $O$ ' rings.

The liquid-gallium pump is an electromagnetic induction pump that uses the rotating magnetic field of the 3-phase winding of an electric induction motor to jenerate the crossed electric current and magnetic fields needed to pump the liquid metal. ${ }^{1}$

The crystals were mounted as shown in Fig. 5. Both the horizontal $(x)$ and vertical $(y)$ positions of both crystals were adjusted with linear translation stages. Rotation stages were used to set the diffraction angles $(\theta)$, and an additional linear translation stage was used to adjust the separation ( $z$ ) between the two crystals. 
For the inclined case, we attached aluminum wedges with a wedge angle of $20^{\circ}$ to the rotation stages. The cooled first crystal was mounted directly on one wedge, and a small motor-driven goniometer with 2 axes was placed between the second crystal and its wedge. One of the axes of this small goniometer corresponds to a crystal tilt adjustment in the flat-crystals case. For the flat-crystals case, the wedges were replaced with ' $L$ ' - shaped brackets.

The optical arrangement at CHESS is shown in Fig. 6 .

\section{Infrared Pyrometry Results}

A photograph made with an infrared camera is shown in Fig. 7. The enlarged footprint in the inclined case completely spans the cooled portion of the crystal. This was expected because the horizontal beam width listed in Table II, namely, $8.0 \mathrm{~mm}$, yields a value of $53 \mathrm{~mm}$ for the length denoted as c in Fig. 2, and the expected length of the long edge of the parallelogram shaped footprint is $57 \mathrm{~mm}$. The vertical height of $4.7 \mathrm{~mm}$ contributes another $11 \mathrm{~mm}$ to the total distance of $68 \mathrm{~mm}$ between acute angles of the parallelogram.

A summary of the pyrometry data for the both the flat and inclined cases are shown in Fig. 8. These temperatures were measured at the hottest point on the beam footprint for a liquid gallium flow rate of 1 gallon per minute. We note that the inclinedcrystal results are significantly cooler than the flat-crystal results. The ratio of flat to inclined case temperatures is roughly 1.8 .

\section{Diffraction Results}

Rocking curves for the flat case are shown in Fig. 9. These were obtained after calibrating the energy of the monochromator ( at $3 \mathrm{~mA} \mathrm{CESR} \mathrm{current)} \mathrm{by} \mathrm{placing} \mathrm{a}$ vanadium filter between the $I_{0}$ and $I_{1}$ ionization chambers and scanning through the $\mathrm{K}$ edge at $5.465 \mathrm{KeV}$. After this step, we ascertained that the spectrum of the angle integrated flux for the first harmonic from the undulator agreed with results obtained in 19897 . The gap of the undulator was set at $1.7 \mathrm{~cm}$, which placed the peak of the first harmonic at $5.14 \mathrm{KeV}$. We then tuned the double-crystal monochromator to $5 \mathrm{KeV}$, placed a $2 \mathrm{~mm}$ thick aluminum filter between the ionization chambers and obtained the rocking curve data. In this arrangement, the $I_{0}$ data are for the (111) reflection at 5 $\mathrm{KeV}$, and the $\mathrm{I}_{1}$ data are for the (333) reflection at $15 \mathrm{KeV}$. The peak values of the (111) rocking curves are plotted versus ring current in Fig. 10. The FWHM values of both the (111) and (333) rocking curves are shown in Fig. 11. The (111) value at the lowest ring current was 17.8 arc sec which equals the ideal self-convolved Darwin-Prins value. The (333) FWHM obtained at the lowest ring current was 4 arc sec which consideratly exceeds the self-convolved Darwin-Prins value of $1.1 \mathrm{arc} \mathrm{sec}$. We conclude that the FWHM of the (333) rocking curves were broadened by a few arc sec for reasons unrelated to the heat load, for example, pressure effects of the gallium flow or mounting strains. We note that thermal broadening set in at 20-30 mA for the (111) and at 10-20 mA for the (333). 
After obtaining the flat-crystals results, we inclined the crystals by inserting wedges as shown in Fig. 5, recalibrated the monochromator, drove to $5 \mathrm{KeV}$ for the $(11 \overline{1})$, and obtained rocking curves at various ring currents. We have found that the simplest way to achieve the desired Bragg diffraction for both crystals in the inclined case is to first center the beam on the first crystal. Then, the downstream distance of the second crystal is set according to the desired beam offset. Lastly, the second crystal is moved in a direction transverse to the beam ( $x 2$ in Fig. 5) until the correct face-to-face distance between the crystals is reached. This face-to-face distance is given by $D \cos \beta / 2 \cos \theta$, where $D$ is the beam offset. The inclined crystal data that we obtained are shown in Figs. 10,12, and 13. Peak values in the inclined case were scaled to lower values with a scaling factor $(0.78)$ determined so that peak values at low currents would be the same for both flat and inclined cases. A dramatic improvement over the flat-crystals case is evident in the inclined-crystals data. Peak values of (11i) rocking curves do not show any evidence of thermal effects up to 60 $\mathrm{mA}$ and continue to increase with increasing ring current up to the highest value of 96 $\mathrm{mA}$. The (111) FWHM values show only a slight broadening from 13 up to 14 arc sec beyond $60 \mathrm{~mA}$, and the (33) FWHM values were not noticably affected. Values for the $(33 \overline{3})$ FWHM were obtained by linear interpolation of the raw data, and they are of relatively low precision. They ranged from 2.5 arc sec at $2 \mathrm{~mA}$ to 2.9 arc sec at $96 \mathrm{~mA}$. The step size for all our data was 0.9 arc sec.

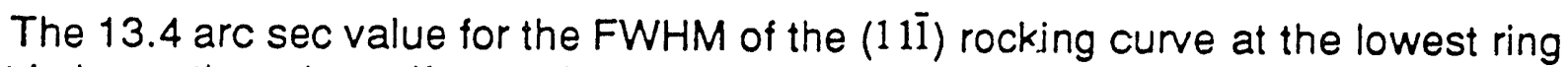
current is lower than the self-convolved Darwin-Prins value of 17.8 arc sec that applies to syminetric diffraction. We have explained this initially rather puzzling observation as resulting from misalignment of either the first or second crystal or, more likely, misalignment of both crystals. In the flat case one has a rotational degree of freedom in the rotation around $\vec{H}$, the reciprocal lattice vector. This azimuthal degree of freedom, which we have called $\rho$, is sacrificed in the inclined case, and an improper setting for $\rho$ can result in trully asymmetric diffraction (i.e., b not equal to -1). Either a broadening or a narrowing can be obtained in this way ${ }^{10}$. We are not able to apportion the net effect of these misalignments to each of the two crystals. However, we can state that if one of the crystals was perfectly set for $b=-1$, then the other was misaligned by $\left|6.7^{\circ}\right|$ in $\rho$ to produce a value of 7.8 or $(7.8)^{-1}$ for $b$. Such a value of $b$ is needed to narrow the (111) Darwin-Prins FWHM for the misaligned crystal from 12.6 arc sec, which is the symmetric $5 \mathrm{KeV}$ (111) value, to $4.5 \mathrm{arc} \mathrm{sec}$. The quadrature addition of 12.6 arc sec for the aligned crystal to 4.5 arc sec for the misaligned crystal yields the observed value of 13.4 arc sec. If the two crystals were equally misaligned by only $\left|2.5^{\circ}\right|$ in $\rho$ to produce values of $(1.8)^{-1}$ for $b$ of the first crystal and 1.8 for $b$ of the second crystal, then the observed result of 13.4 arc sec would have resulted from the quadrature addition of 9.5 arc sec and 9.5 arc sec. A situation with more equal misalignments was more likely. Thus goniometers that permit $p$ adjustments (which we did not have) are highly desirable. 
We observed the shape of the beam after the second crystal, and, in the inclined case, we found that it was angled out of the horizontal plane. In the inclined geometry, the beam is spread out by the first crystal, and, ideally, it can be brought back to its original shape with an identical second crystal that exactly undoes the effect of the first crystal. However, if the two crystals are not closely matched, the beam exiting the whole monochromator will have an altered cross section. In the case of flatcrystals, this effect is very weak because of a low sensitivity to the exact cut of the surface. However for an inclined-crystal monochromator with a large angle of inclination the sensitivity to the exact cut of the surface is greatly enhanced. In the present case the diffracting surfaces of the two crystals were individually formed. In retrospect, a better way would have been to use opposing faces from a single saw cut.

\section{Summary}

We have demonstrated that an inclined-crystal monochromator results in a dramatically improved performance compared to a standard monochromator. Rocking curves obtained with 379 Watts total power and a peak power density of $48 \mathrm{Watts} / \mathrm{mm}^{2}$ showed a decrease in peak value of only $12 \%$ and an increase in FWHM of only 1 to 2 arc sec due to thermally induced distortions.

Acknowledgements

We are indebted to Prof. B. Batterman and Dr. D. Bilderback and the entire CHESS staff for their help and assistance. This work was supported by the U.S. Department of Energy, BES-Materials Science under contract No. W-31-109-ENG-38. 


\section{FIGURE CAPTIONS}

1) The inclined-crystal geometry. The top portion shows a perspective view. The value of $\beta$, the angle between the (111) surface and the (11i) Bragg planes, is $70.529^{\circ}$.

2) The footprint on the crystal surface in the inclined geometry resulting from a horizontal beam having a rectangular cross section. The top portion is a perspective view.

3) Calculated power density profile for the present experiments.

4) The silicon crystal and stainless steel mount. The crystal was made by cementing a grooved top piece to a second piece of silicon. The cemented assembly was clamped to a stainless steel base and sealed with ' $O$ '-rings.

5) The monochromator viewed from an upstream vantage point in two configurations: a) standard flat crystals; b) inclined crystals. Both configurations are shown at zero Bragg angle, i.e., for $\theta_{1}=\theta_{2}=0$.

6) Schematic layout of the beamline components.

7) Infrared camera image of the first crystal in the inclined arrangement. The footprint of the beam is seen as a stripe. One corner of the second crystal holder is also visible as is its reflection in the first crystal.

8) Temperature difference between the hottest point of the beam footprint and the surrounding cooled portion of the crystal. The pyrometry measurements were made with an infrared sensitive camera.

9) Rocking curves obtained in the standard flat-crystals arrangement.

10) Peak rocking curve intensities versus CESR current. A least squares linear fit to the inclined case data for currents $\leq 50 \mathrm{~mA}$ is also shown.

11) FWHM values for rocking curves in the flat-crystals case.

12) Rocking curves obtained in the inclined-crystal arrangement.

13) FWHM values for rocking curves in the inclined-crystals case. 
' R.K. Smither, G.A. Forster, C.A. Kot, and T.M. Kuzay, Nuclear Instruments and Methods A266, 517 (1988).

${ }^{2}$ P. van Zuylen, A.D. Lemaire. A.J.Th.M. Wijman, Memorandum No. 832.020, Technisch Physische Dienst TNO-TH, Stieltjesweg 1, Deltt, Netherlands, July 1988.

${ }^{3}$ P.L. Cowan and S. Brennan, Rev. Sci. Instrumen. 60, 1987 (1989).

4W.K. Lee, A.Macrander, D. Mills, R. Smither, and A. Khounsary, Workshop on Thermal Problems of Synchrotron Radiation Optics, held as a satellite meeting to the Intemational Synchrotron Radiation Instrumentation Meeting, July 20, 1991, Chester College, UK.

${ }^{5}$ A. Khounsary, Proc. of 4th Int. Conf. on Synchrotron Radiation Instrumentation, Rev. Sci. Instrum. in press.

- J. Hrdy, Proc. of 4th Int. Conf. on Synchrotron Radiation Instrumentation, Rev. Sci. Instrum., in press.

7 D.H. Bilderback, B.W. Batterman, M.J. Bedzyk, K. Finkelstein, C. Henderson, A. Merlini, W.

Schildkamp, Q. Shen, J. White, E.B. Blum, P.J. Viccarro, D.M. Mills, S. Kim, G.K. Shenoy, K.E. Robinson, F.E. James, and J.M. Slater, Rev. Sci. Instrum. 60, 1419 (1989).

B.W. Batterman and H. Cole, Rev. Mod. Phys. 36, 681 (1964)

- W.H. Zachariasen, Theory of X-ray Diffraction in Crystals, (Dover, New York 1967)., Chap.3.

10 A.T. Macrander and W.K. Lee, these Proceedings

"Kwang-Je Kim, AIP Conference Froceedings 184, Vol.1, 1989, p.565.

12 R.K. Smither, W.K.Lee, A.T. Macrander, D.M. Mills, and C.S. Rogers, Proc. of 4th Int. Conf. on Synchrotion Radiation Instrumentation, Rev. Sci. Instrum., in press.

${ }^{13}$ R.K. Smither, G.A. Forster, D.H. Bilderback, M. Bedzyk, K. Finkelstein, C. Henderson, J. White, L.E.Berman, P. Stefan, and T. Oversluizen, Rev. Sci. Instrumen. 60, 1486 (1989). 


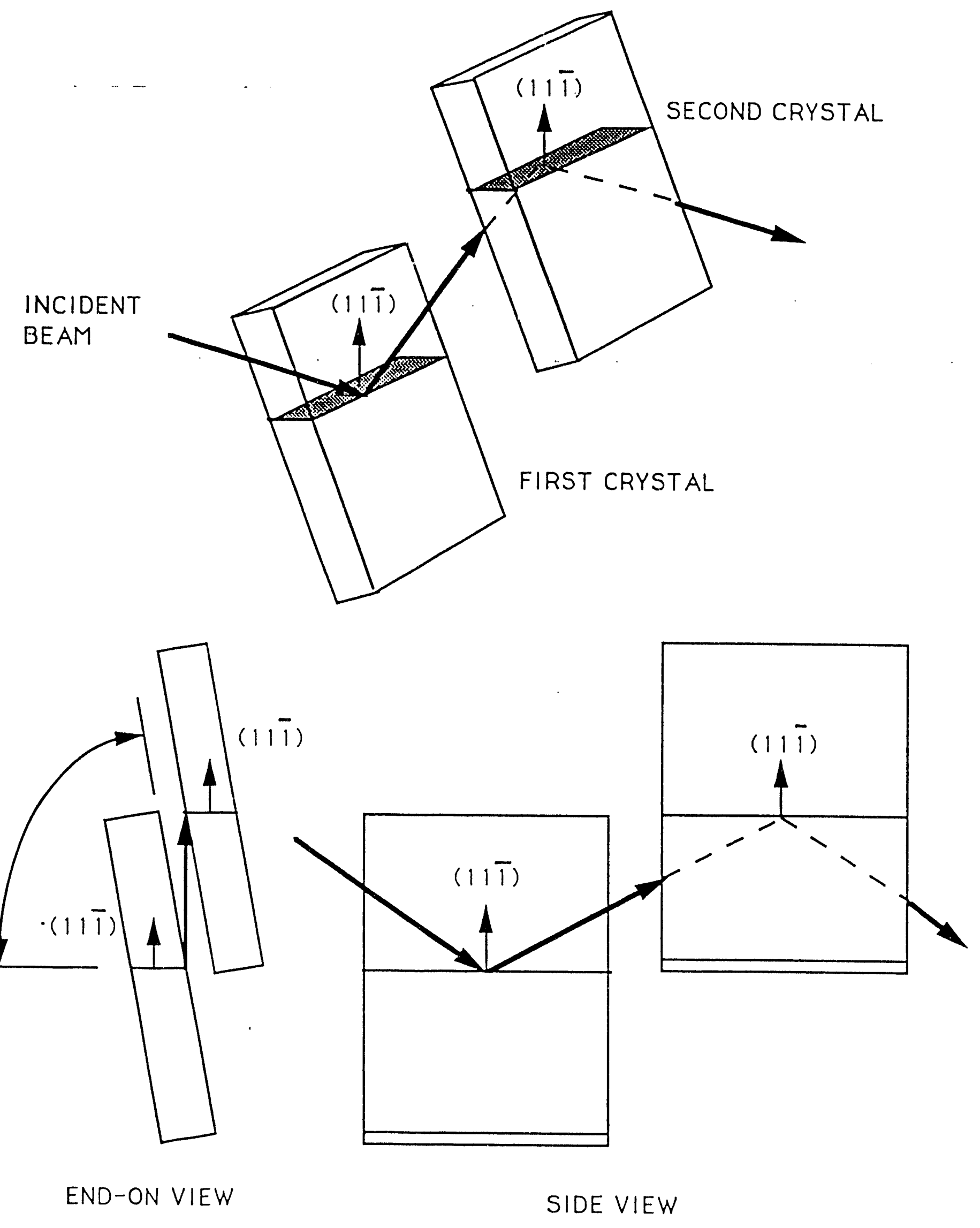



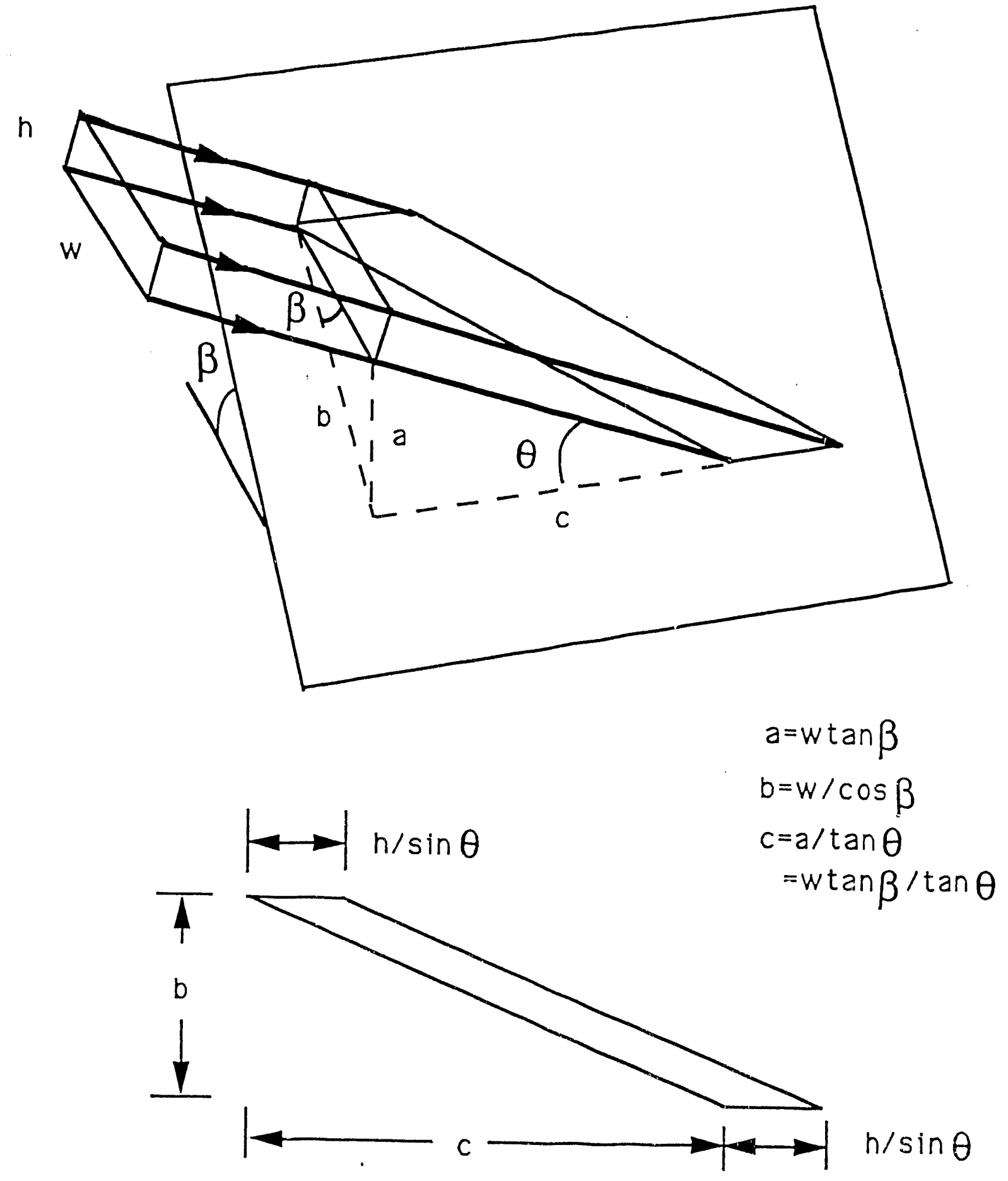
POWER DENSITY OF APS/CHESS UNDULATOR - RING ENERGY $=5.43 \mathrm{GeV}$

$G A P=1.7 \mathrm{~cm}$

$100 \mathrm{~mA}$

DISTANCE FROM UNDULATOR $=18 \mathrm{~m}$

TOTAL POWER AT CRYSTAL $=3.95 \mathrm{~W}$

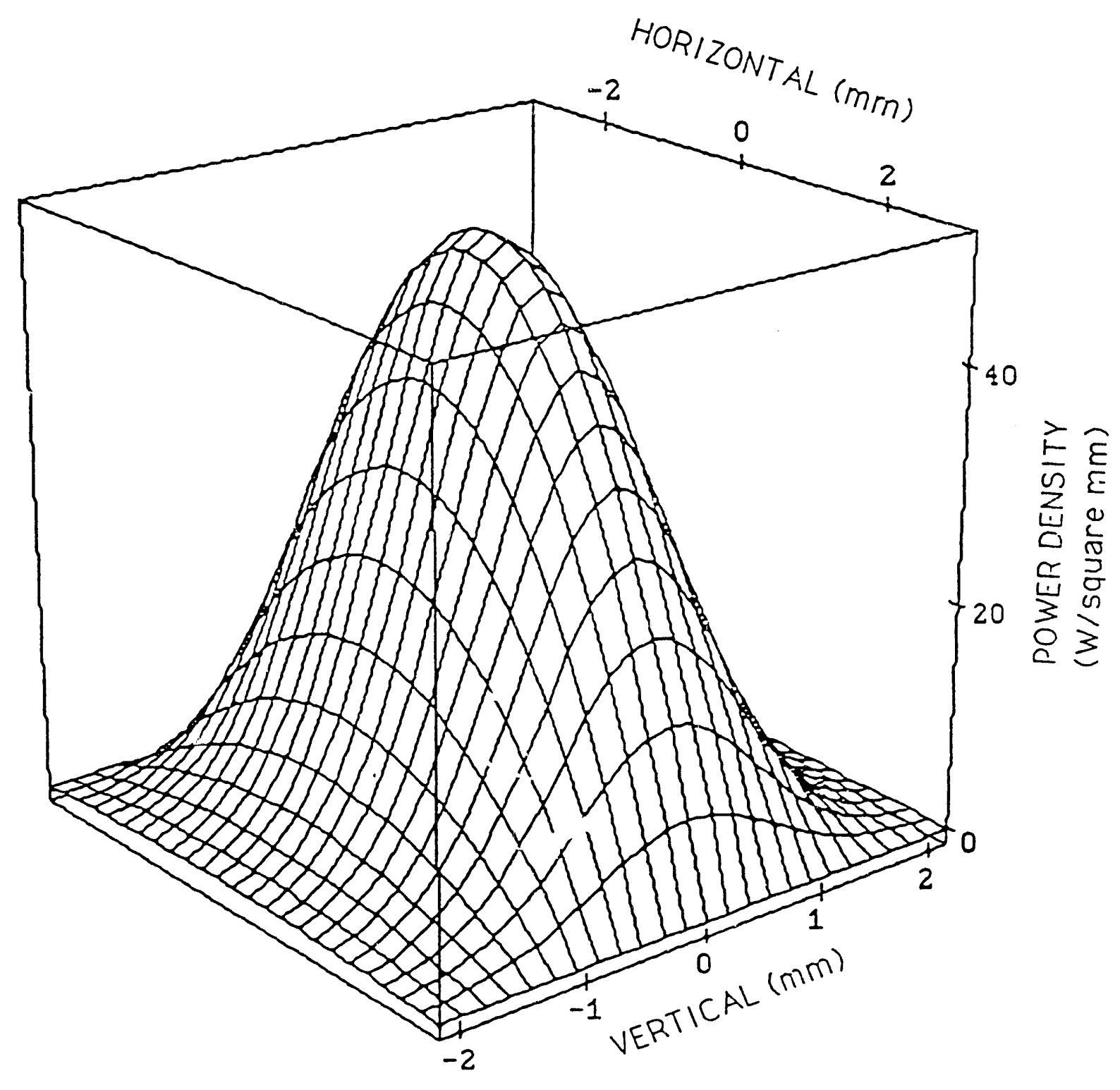




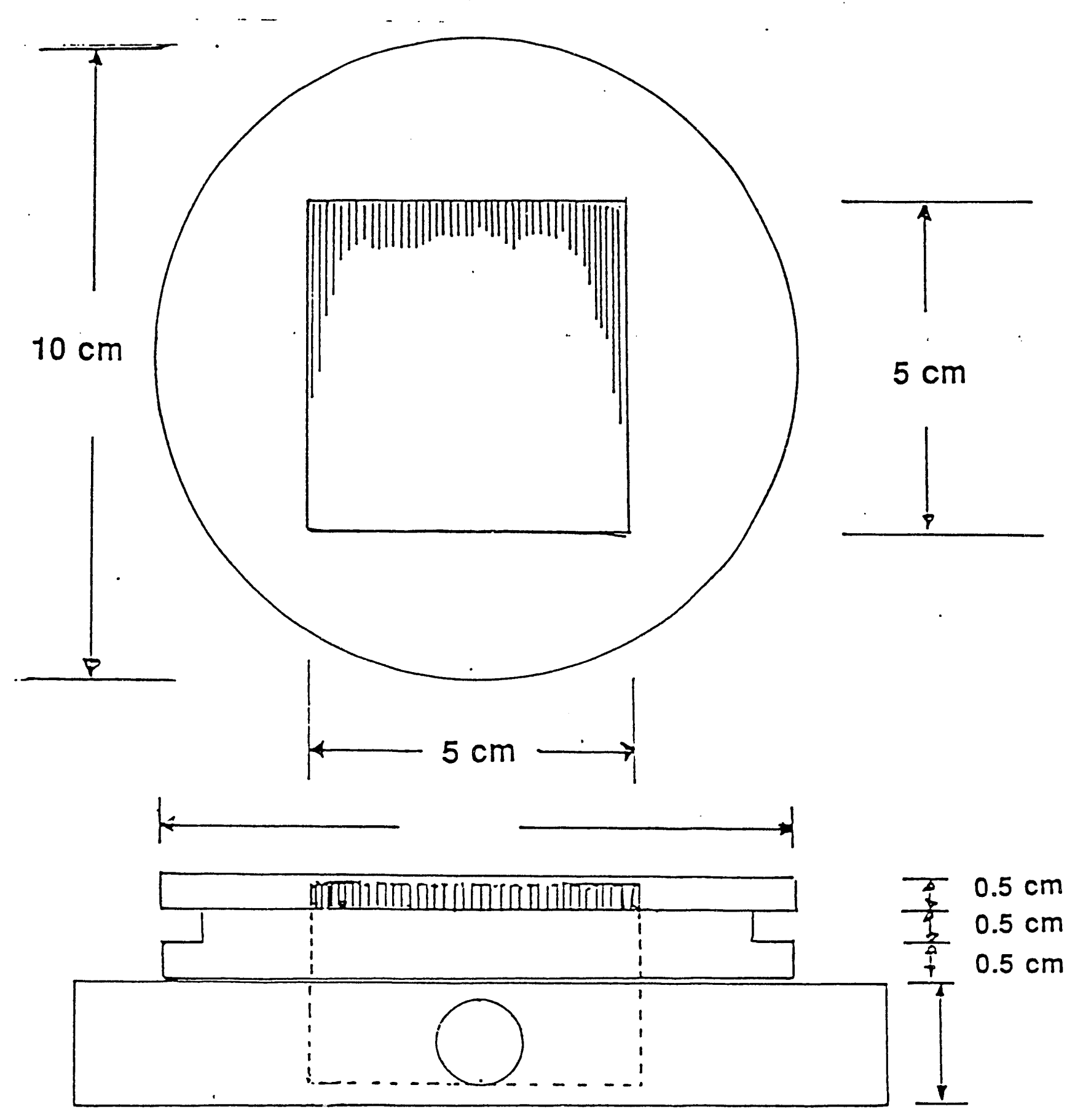



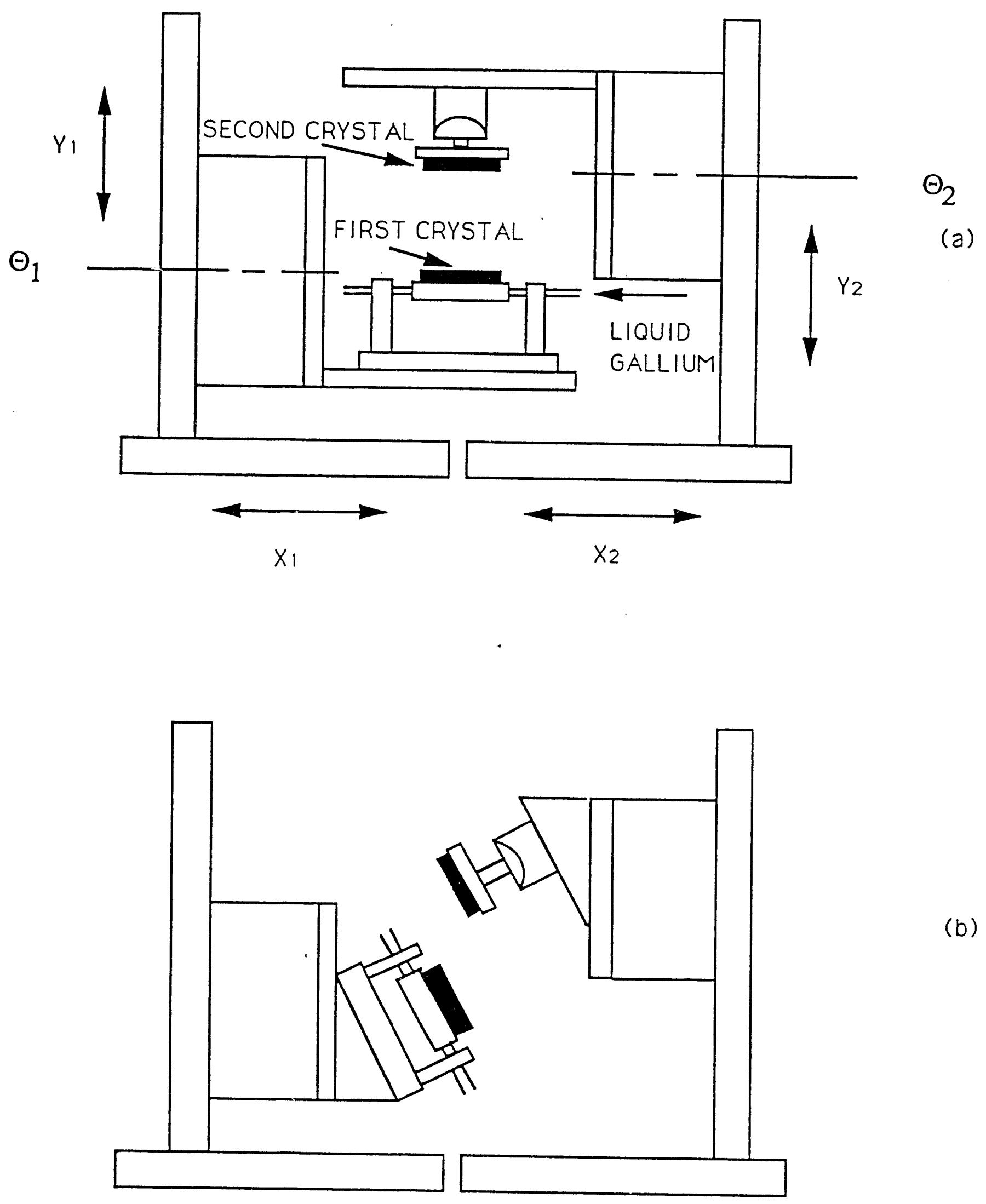

(b) 


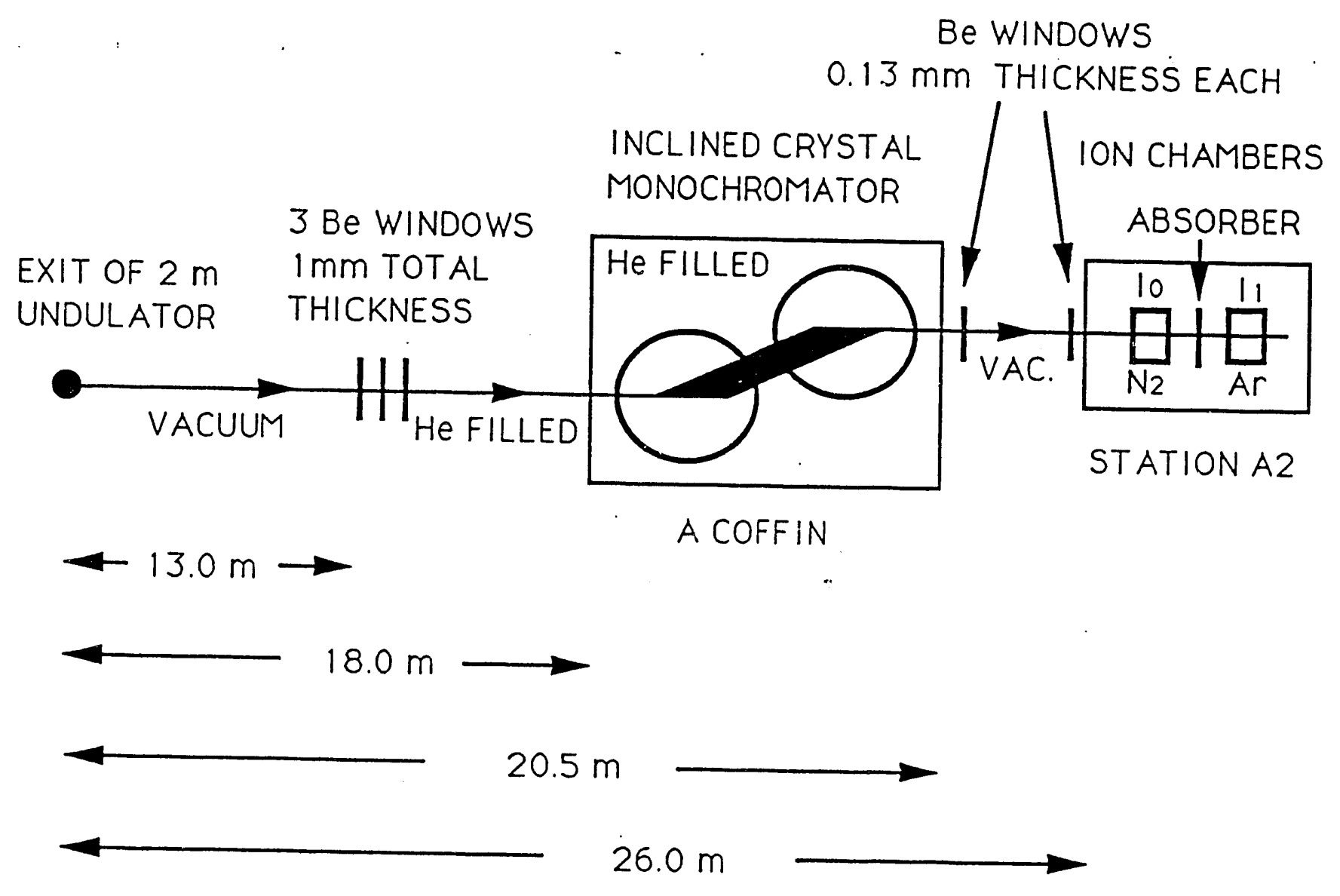




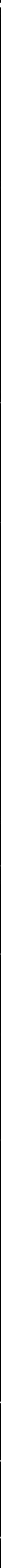




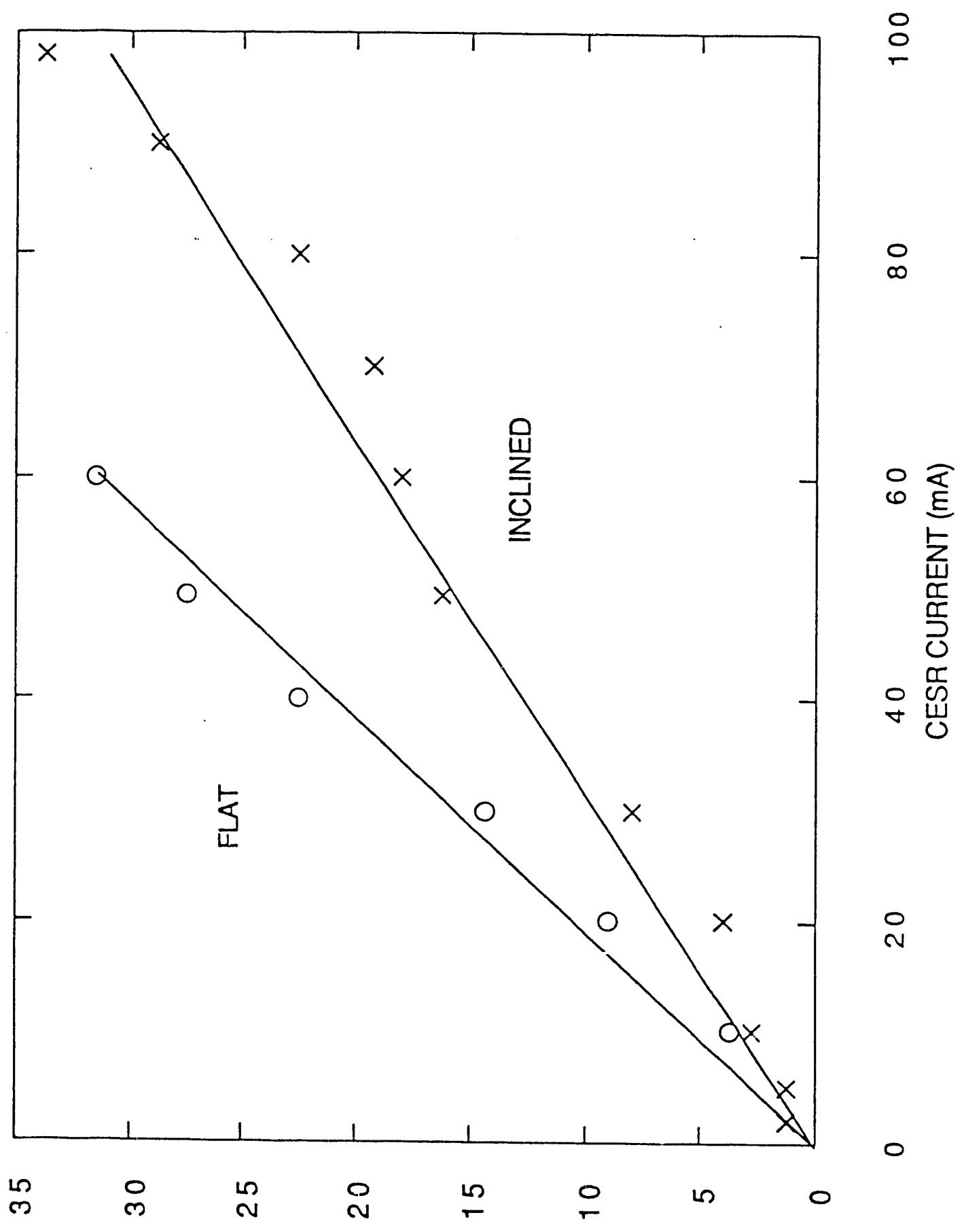

(๖ヨ๐) $\exists$ SI $\exists \forall \cap \perp \forall y \exists d W \exists \perp$ 


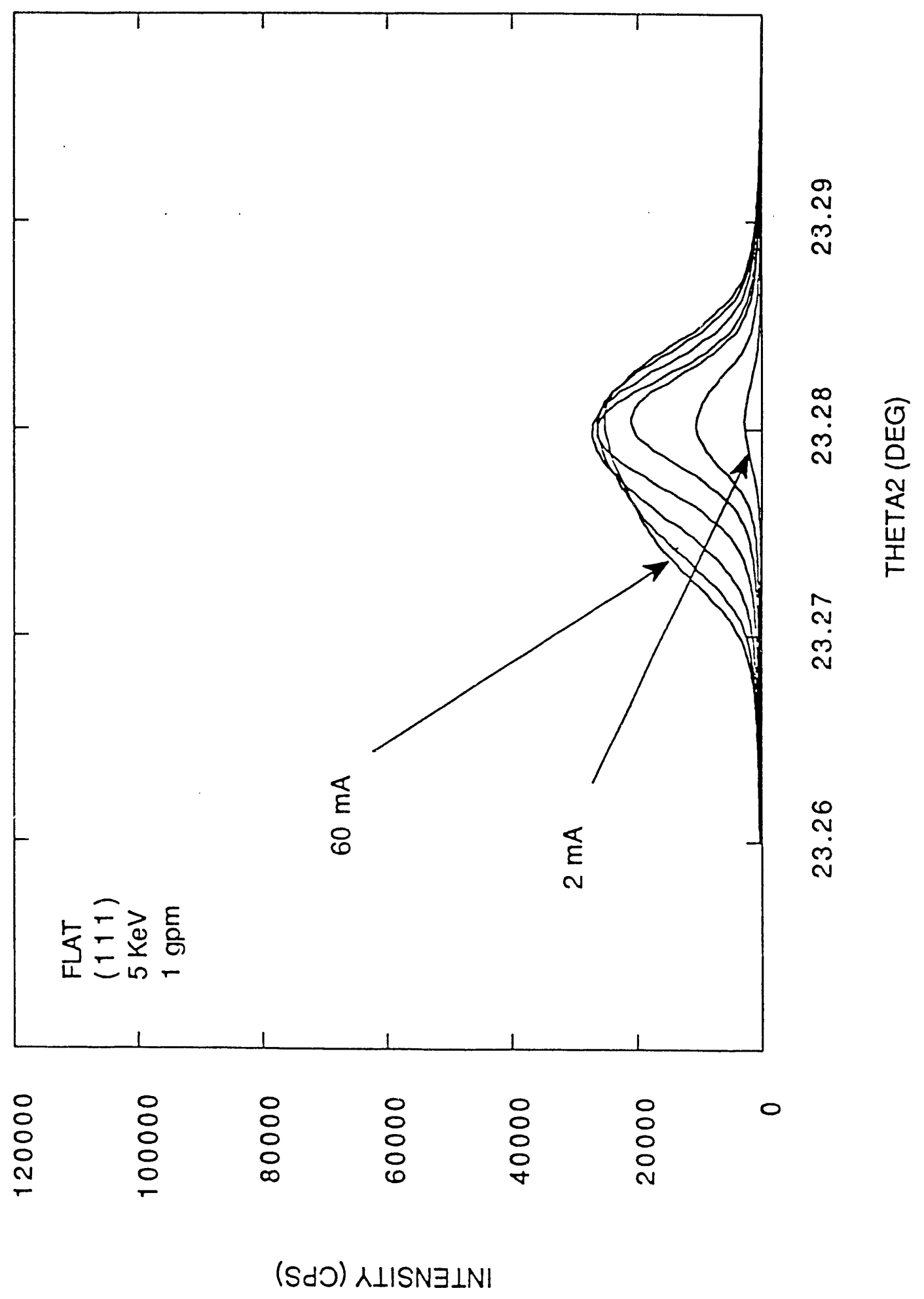




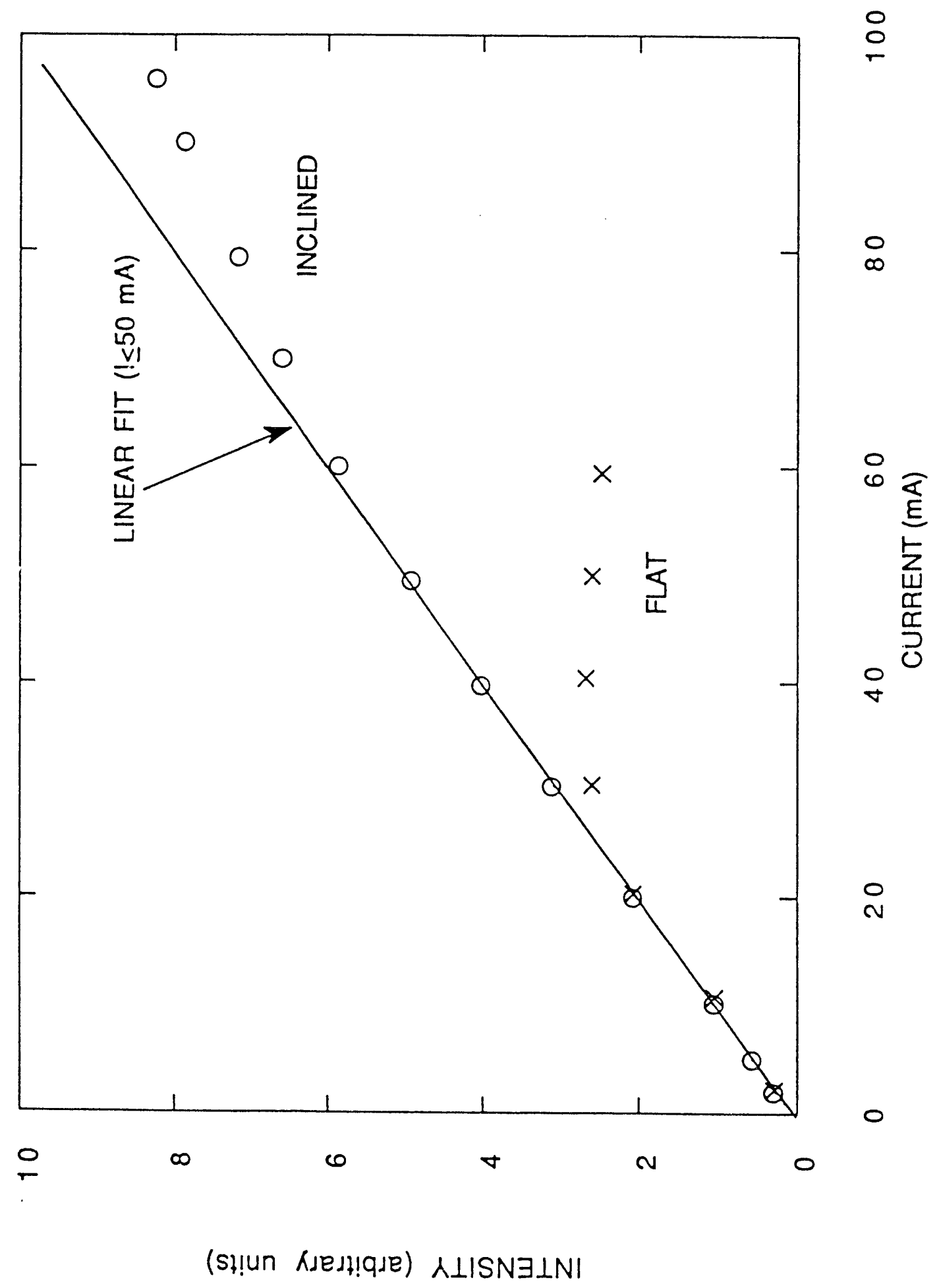




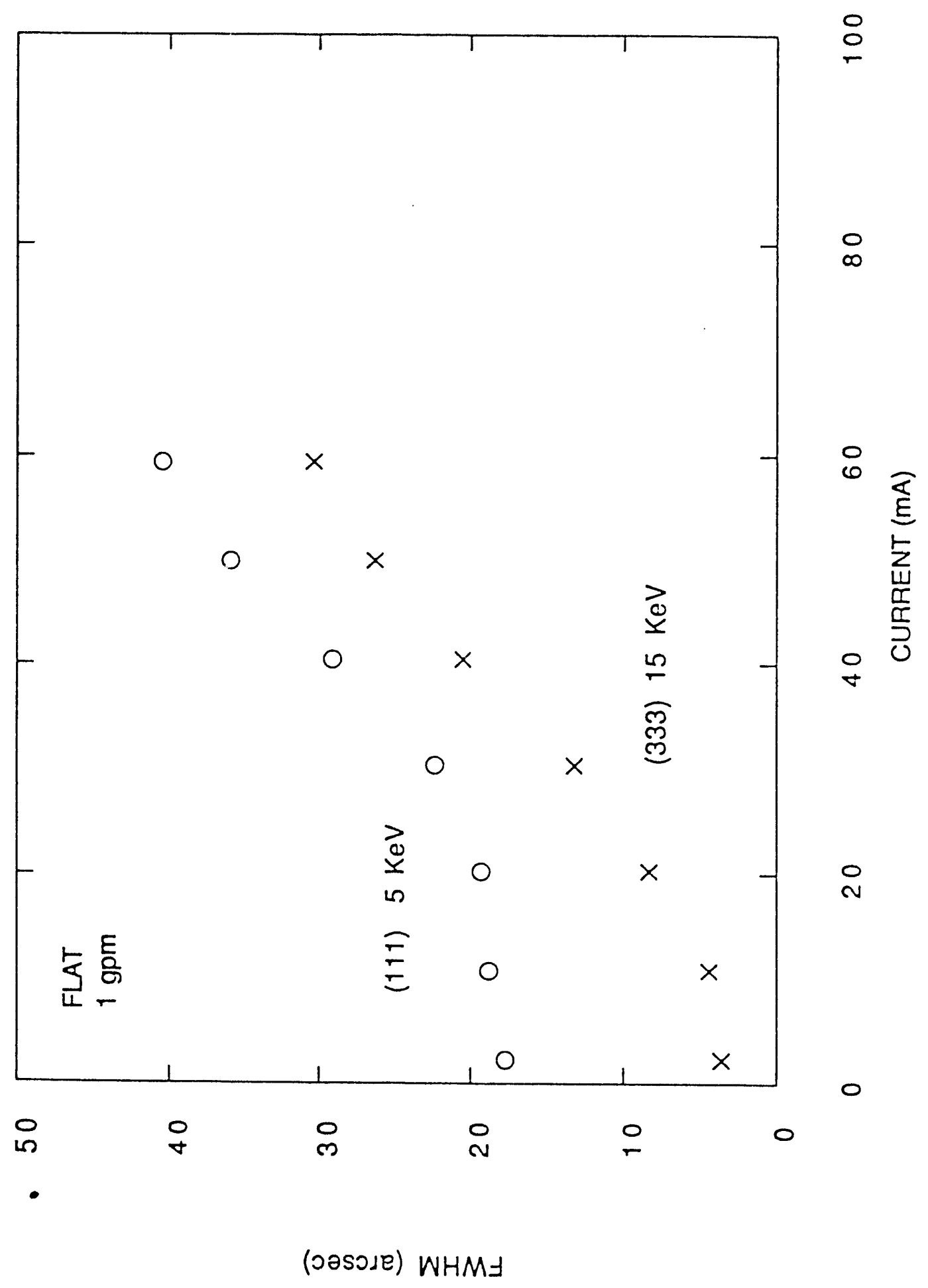




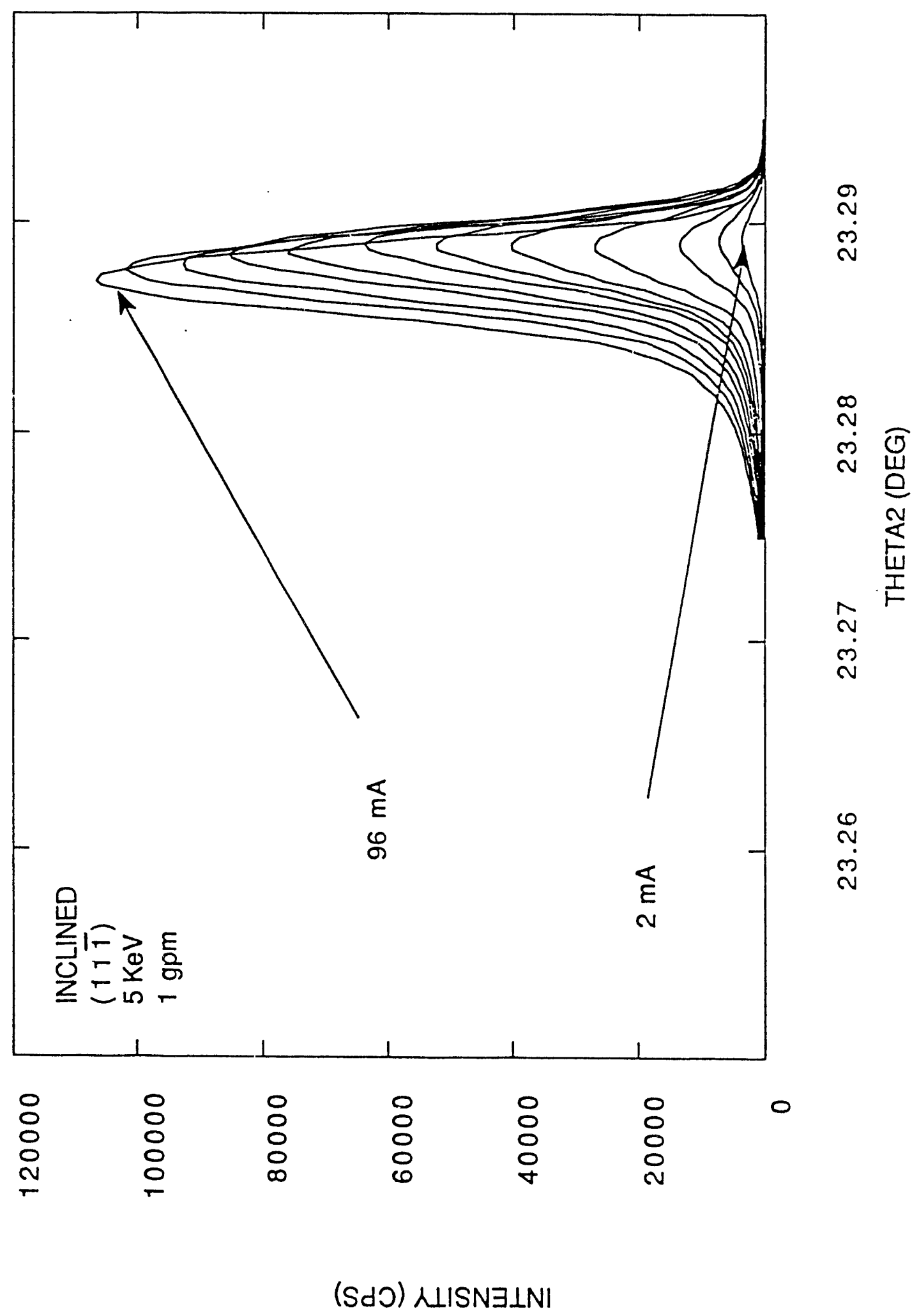




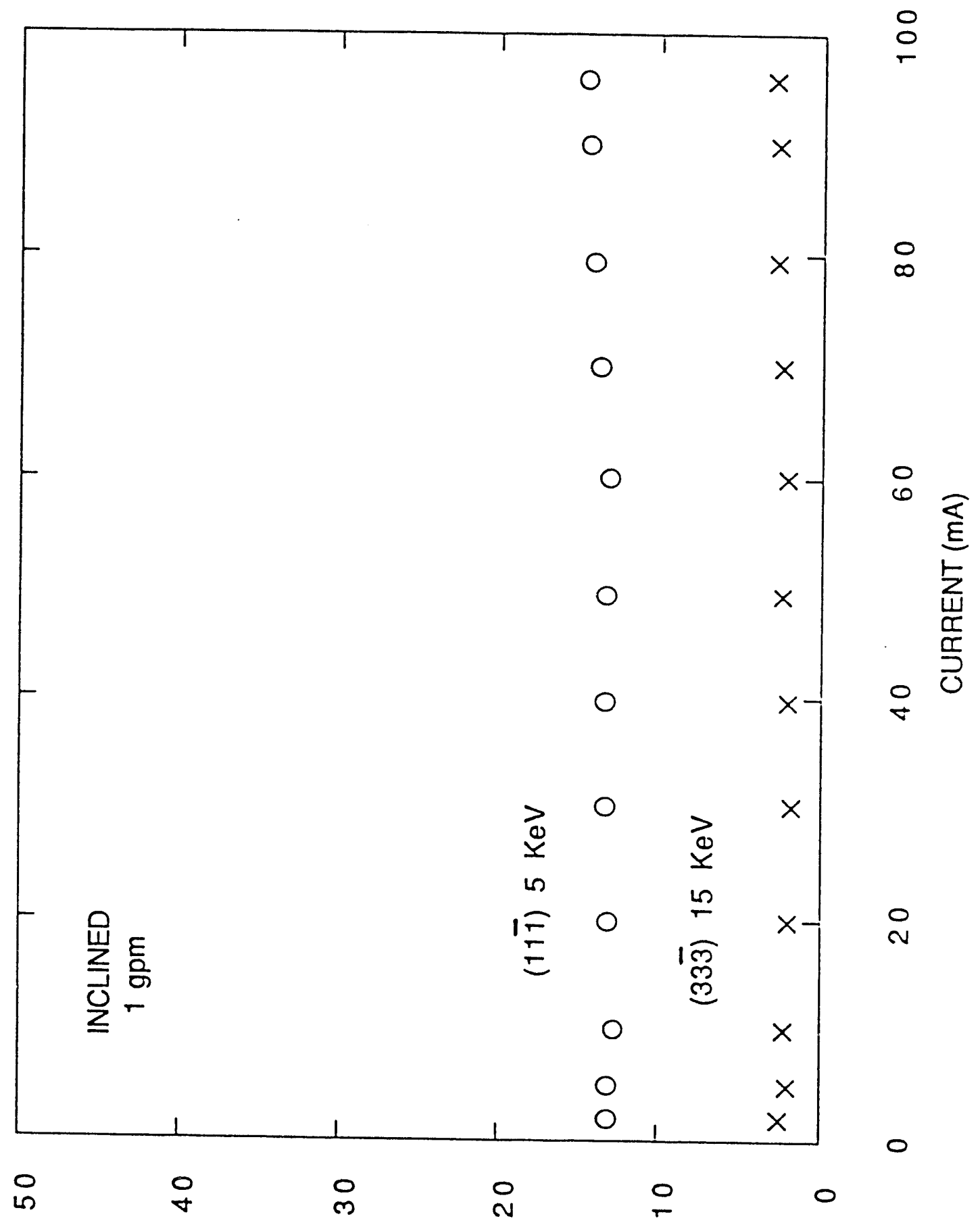

(Oasode) WHMJ 

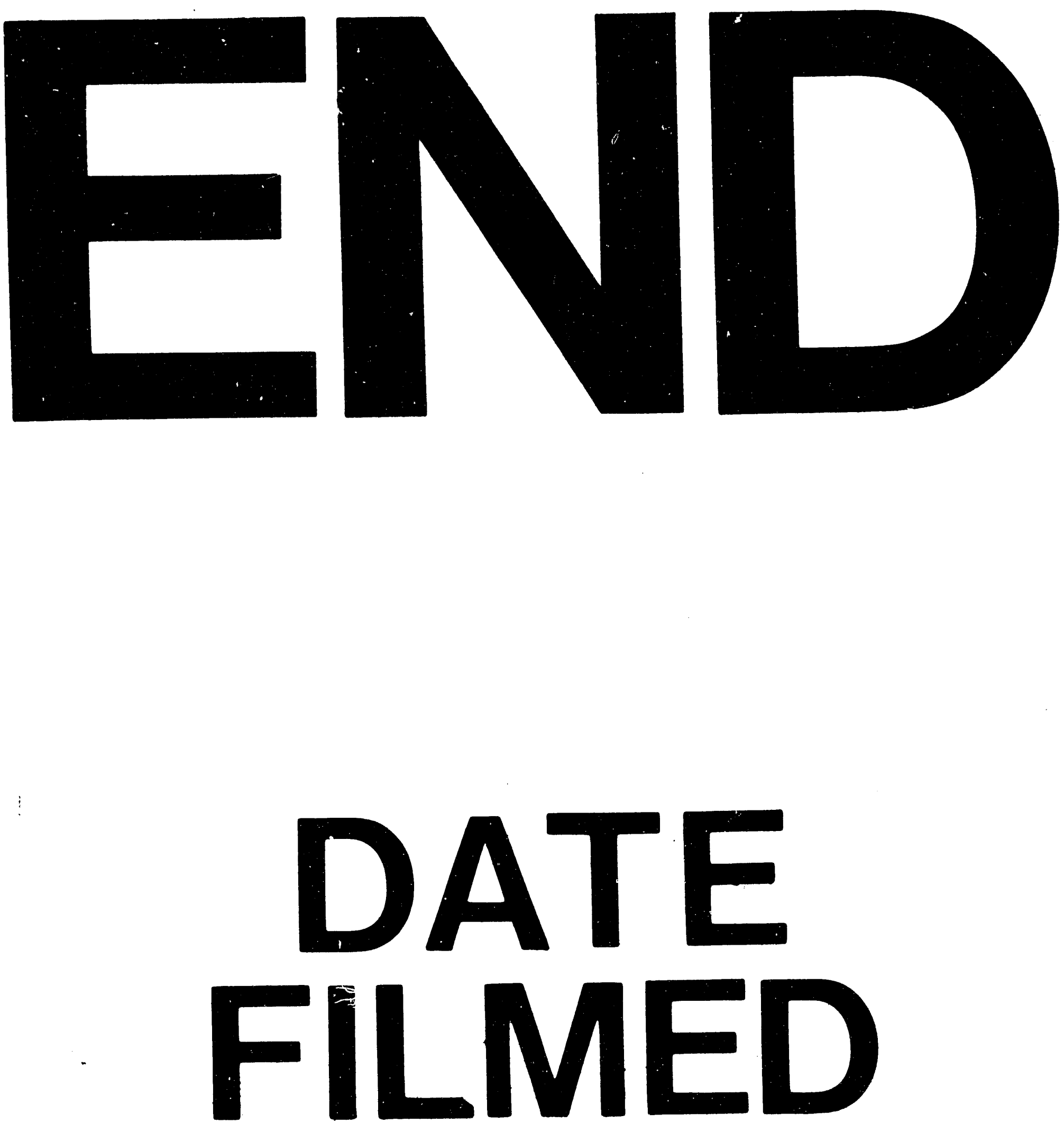

I

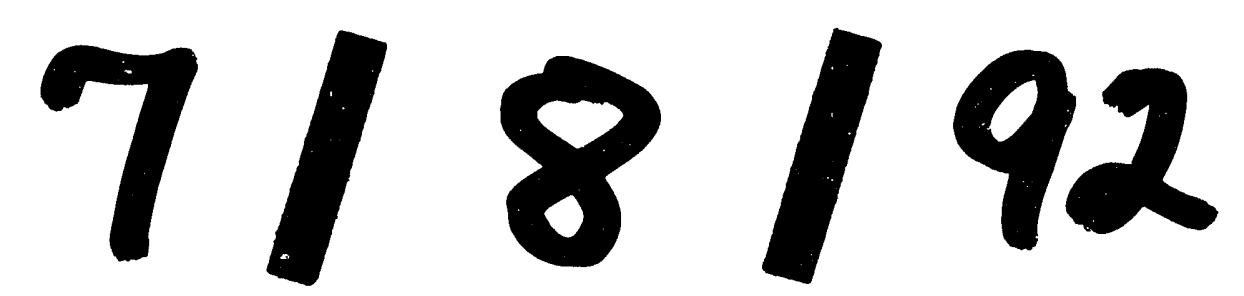




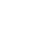

